\title{
RADIOMETRIC DATING OF IGNEOUS ROCKS FROM SIERRAS PAMPEANAS, ARGENTINA
}

\author{
RAFAEL R. GONZÁLEZ* e ALEJANDRO J. TOSELLI*
}

\begin{abstract}
In this paper new radiometric dates on igneous rocks (K-Ar method) are given. They confirm the threefold subdivision in the major areas of Sierras Pampeanas, suggested by Linares and La Torre for paleozoic magmatic cycles in the provinces of Cordoba and San Luis; the older cycle during Cambro-Ordovician times, the intermediate during the Devonian, and the younger one during the Carboniterous.
\end{abstract}

RESUMO Novos dados radiométricos obtidos pelo método K-Ar, permitem que seja reconhecida divisão tríplice no Paleozóco das Sierras Pampeanas da Argentina, correspondente aos ciclos magmáticos datados por Linares e La Torre. O primeiro deles ocorre no Cambro-Ordoviciano e o segundo, no Carbonífero. Entre eles outro ciclo Devoniano também ocorre.

INTRODUCTION During the last twenty-five years, some scientists (Harrington, 1956; González Bonorino, 1950 and others) considered both metamorphic and intrusive rocks outcropping in the whole area of Sierras Pampeanas as Precambrian in age. However, many years before, Rassmuss (1918) and Bonarelli and Pastore (1918), divided without field evidences the magmatic activity in two cycles, one of Precambrian age, the other, Paleozoic (Devonian). This opinion was flung up until radiometric dates re-opened the problem.

Kittl (1965), Linares (1968), Stipanicic and Linares (1969), Linares and La Torre (1969) and Halpern et al. (1970) exhibit radiometric dating of some igneous rocks from several localities of Sierras Pampeanas. The results could not establish a Precambrian activity, although the results obtained between 500-550 m.y. (K-Ar method) might suggest that the rocks could be Precambrian in age. Moreover, metamorphic basement dates (Rb-Sr method) given by Halpern et al. (1970) offer lower Paleozoic ages, specially reported from the southern hills in Córdoba and San Luis Provinces. Three magmatic cycles were then determined: 1st. between $450-520$ m.y.; 2nd. corresponding to 350-380 m.y. and 3rd., the younger, 300-330 m.y. old.

Later on, González (1971) and González et al. (1971) determined the chronological position of additional granitic rocks in some distant places of the Sierras Pampeanas, as Sierra de Ancasti, Sierra de Velasco and Cumbres Calchaquíes. Dates given by' Kittl (1965) for granites of San Luis and by Caelles (1971) for similar rocks in Catamarca are also suggestive.

In more recent times, the present authors analyzed several samples from other localities such as the southwestern hills of the Province of La Kioja (Sierras de Los Llanos, Chepes and Malanzán), the southeastern slope of the Sierra de Aconquija (Province of Tucumán) and so made M. Cabrera (personal communication) in Sierra del Cajón (Province of Catamarca). All the new results confirm the ideas of Linares and La Torre about the Paleozoic magmatic cycles.

RESULTS AND DISCUSSION In Table I the ages of rocks from several localities are listed; the samples dated by the authors are marked with $\left(^{*}\right)$. The order of tabulation shown

* Instituto Miguel Lillo - Universidad Nacional de Tucumán, Argentina 
gives the values from the youngest to the oldest rocks. In the author's opinion, it is possible to group the results as follows:

1) An old magmatic cycle between $540-400$ m.y.;

2) An intermediate cycle between $395-350$ m.y., and

3) A younger one between $345-330 \mathrm{~m} . \mathrm{y}$.

Table 1 - Radiometric ages of the igneous rocks

\begin{tabular}{|c|c|c|c|c|c|c|}
\hline Sample & Description and Locality & Method & Mineral & Age in m.y. & Laboratory & Reference \\
\hline LZZ-001 & Porphyritic granite, Bialet Massé, Cba & $\mathrm{Pb} / \mathrm{a}$ & zircón & $246 \pm 25$ & 3 & 3.4 \\
\hline LZ-019 & Granite, Km 83-Pampa de Achala-Cba & $\mathrm{Ar} / \mathrm{K}$ & biotite & $250 \pm 10$ & 2 & 4 \\
\hline \multirow[t]{3}{*}{$\begin{array}{l}L Z-001 \\
L Z-128\end{array}$} & $\begin{array}{l}\text { Porphyritic granite, Bialet Massé, Cba } \\
\text { Granite - Cantera Giobelli, La Toma, San }\end{array}$ & $\mathrm{Ar} / \mathrm{K}$ & muscovite & $301 \pm 10$ & 2 & 4 \\
\hline & Luis & $\mathrm{Ar} / \mathrm{K}$ & & $303 \pm ?$ & 5 & 4 \\
\hline & Granite - La Toma, San Luis & $\mathrm{Ar} / \mathrm{K}$ & & $303 \pm ?$ & 5 & 1 \\
\hline 2132 & Granite - Rio Arenal, Aconquija, Tuc. & $\mathrm{Ar} / \mathrm{K}$ & biotite & $305 \pm 10$ & 1 & $(*)$ \\
\hline $\mathrm{LZ} \cdot 008$ & Porphyritic granite - Capilla del Monte, Cba & $\mathrm{Ar} / \mathrm{K}$ & muscovite & $307 \pm 10$ & 2 & 4 \\
\hline AC-SI 3 & Granite - San Ignacio - Aconquija, Tuc. & $\mathrm{Ar} / \mathrm{K}$ & muscovite & $310 \pm 15$ & 2 & $(*)$ \\
\hline $\mathrm{AC}-\mathrm{S} 13$ & Granite - San Ignacio - Aconquija, Tuc. & $\mathrm{Ar} / \mathrm{K}$ & biotite & $319 \pm 10$ & 2 & $(*)$ \\
\hline 2132 & Granite - Rio Arenal - Aconquija, Tuc. & Ar $/ \mathrm{K}$ & muscovite & $320 \pm 9$ & 1 & $(*)$ \\
\hline AC-LP5 & Granite - Los Pinos - Aconquija, Tuc. & $\mathrm{Ar} / \mathrm{K}$ & biotite & $321 \pm 10$ & 2 & $(*)$ \\
\hline LZ-055 & Porphyritic granite - Capilla del Monte, Cba & $\mathrm{Sr} / \mathrm{Rb}$ & whole rock & $325 \pm 25$ & 4 & 4 \\
\hline LZ-001 B & Aplitic granit-Bialet Massé, Cba & $\mathrm{Ar} / \mathrm{K}$ & muscovite & $328 \pm 15$ & 2 & 4 \\
\hline LG-S01 & Porphyritic granite - Los Gigantes, Cba & $\mathrm{Ar} / \mathrm{K}$ & muscovite & $329 \pm 15$ & 2 & 4 \\
\hline LZ-001 & Porphyritic granite - Bialet Masse, Cba & $\mathrm{Sr} / \mathrm{Rb}$ & whole rock & $330 \pm 25$ & 4 & 4 \\
\hline LZZ-012 & $\begin{array}{l}\text { Granite-Rio Yuspe, in the way from Tanti } \\
\text { to Taninga, Cba }\end{array}$ & $\mathrm{Sr} / \mathrm{Rb}$ & whole rock & $330 \pm 25$ & 4 & 4 \\
\hline LZ-019 & Granite - Pampa de Achala way Cba & $\mathrm{Sr} / \mathrm{Rb}$ & whole rock & $330 \pm 25$ & 4 & 4 \\
\hline $\mathrm{LZ}-064$ & Porphyritic granite - $\mathrm{A}^{\circ}$ Las Mojarras, Cba & $\mathrm{Sr} / \mathrm{Rb}$ & whole rock & $330 \pm 25$ & 4 & 4 \\
\hline LZZ-OKG & Granite-Km 72, Pampa de Achala way, $\mathrm{Cba}$ & $\mathrm{Sr} / \mathrm{Rb}$ & whole rock & $330 \pm 25$ & 4 & 4 \\
\hline LZ-068 & $\begin{array}{l}\text { Granite - Km } 108 \text { - Pampa de Achala way, } \\
\text { Cba }\end{array}$ & $\mathrm{Sr} / \mathbf{R b}$ & whole rock & $330 \pm 25$ & 4 & 4 \\
\hline $\mathrm{AC}(\mathrm{LAJ}) 2$ & $\begin{array}{l}\text { Granite - Cumbre Las Lajas, S. } \\
\text { Aconquija }\end{array}$ & $\mathrm{Ar} / \mathrm{K}$ & muscovite & $330 \pm 10$ & 2 & $(*)$ \\
\hline \multirow[t]{2}{*}{ LZ -032} & $\begin{array}{l}\text { Porphyritic granite - Characato, Cba } \\
\text { Granite - La Posta, Pampa de Achala way, }\end{array}$ & $\mathrm{Ar} / \mathrm{K}$ & muscovito & $331 \pm 15$ & 2 & 4 \\
\hline & $\mathrm{Cba}$ & $\mathrm{Pb} / a$ & zircon & $332 \pm 30$ & 3 & 4 \\
\hline \multirow[t]{2}{*}{ LC-VOI } & Ore vein - Los Condores, Concarán, San & & & & & \\
\hline & Luis & $\mathrm{Ar} / \mathrm{K}$ & muscovite & $334 \pm 10$ & 2 & 4 \\
\hline 121 & Granite - Volcán, San Luis & $\mathrm{Sr} / \mathrm{Rb}$ & whole rock & $338 \pm 15$ & 2 & 2 \\
\hline 128 & Granodiorite - La Totora, San Luis & $\mathrm{Sr} / \mathrm{Rb}$ & whole rock & $338 \pm 15$ & 2 & 2 \\
\hline AC-LP4 & Granite - Los Pinos, Aconquija, Tuc. & $\mathrm{Ar} / \mathrm{K}$ & muscovite & $338 \pm 10$ & 2 & $(*)$ \\
\hline \multirow[t]{2}{*}{$\begin{array}{l}\mathrm{VQ}(\mathrm{CPB}) 2 \\
\mathrm{LZ}-020\end{array}$} & $\begin{array}{l}\text { Granite-Campo Pampa Belen - } \mathrm{S} \text {. Vinquis } \\
\text { Granite - Km } 20 \mathrm{~W} \text { from Icho Cruz Pampa }\end{array}$ & $\mathrm{Ar} / \mathrm{K}$ & biotite & $339 \pm 10$ & 2 & $(*)$ \\
\hline & de Achala, Cba & $\mathrm{Ar} / \mathrm{K}$ & muscovite & $345 \pm 15$ & 2 . & 4 \\
\hline LP.ECl & Pegmatite - El Criollo, Tanti, Cba & $\mathrm{Ar} / \mathrm{K}$ & muscovite & $350 \pm 30$ & 6 & 4 \\
\hline LP-SJ1 & Pegmatite - San José - El Mallin Cba & $\mathrm{Ar} / \mathrm{K}$ & biotite & $352 \pm 25$ & 2 & 4 \\
\hline $\mathrm{Z}-1$ & Porphyritic granite - Cuesta de Zapata - Cat. & $\mathrm{Ar} / \mathrm{K}$ & biotite & $355 \pm 15$ & 1 & $(*)$ \\
\hline $\mathrm{AC}-\mathrm{S} 12$ & Granite - San Ignacio, Aconquija, Tuc. & $\mathrm{Ar} / \mathrm{K}$ & muscovite & $359 \pm 15$ & 2 & $(*)$ \\
\hline $\mathrm{Lz}-043$ & Diorite - Las Tunas, Cosquín, Cba & $\mathrm{Ar} / \mathrm{K}$ & biotite & $368 \pm 15$ & 2 & 4 \\
\hline LZ-061. & Tonalite - El Diquesite - La Calera, Cba & $\mathbf{A r} / \mathrm{K}$ & biotite & $370 \pm 25$ & 6 & 4 \\
\hline $\mathrm{SC}(21)$ & Granodiorite - Tres Cerritos, S. del Cajón & $\mathrm{Ar} / \mathrm{K}$ & biotite & $373 \pm 10$ & 2 & $(*)$ \\
\hline LZ-009 & Porphyritic granite - Capilla del Monte, Cba & $\mathrm{Pb} / \mathrm{a}$ & zircon & $373 \pm 45$ & 3 & 4 \\
\hline \multirow[t]{2}{*}{ LN-11 } & Granite - Tama Costa del Medio, Los & & & & & \\
\hline & Llanos, La Rioja & $\mathrm{Ar} / \mathrm{K}$ & biotite & $376 \pm 9$ & 1 & $(*)$ \\
\hline 41 & Pegmatite - Cerro Valdivia - San Juan & $\mathrm{Ar} / \mathrm{K}$ & & $379 \pm ?$ & 7 & 4 \\
\hline 40 & Porphyritic granite - Capilla del Monte, Cba & $\mathrm{Pb} / \mathrm{a}$ & & $379 \pm 40$ & 3 & 3 \\
\hline LZ-008 & Porphyritic granite - Capilia del Monte, Cba & $\mathrm{Pb} / \mathrm{a}$ & zircon & $380 \pm 46$ & 3 & 4 \\
\hline $\mathrm{LN}-22$ & Tonalite - Chelco - S de Chepes, La Rioja & $\mathrm{Ar} / \mathrm{K}$ & biotite & $381 \pm 20$ & 1 & $(*)$ \\
\hline $\mathrm{BE}-1$ & Granite - Sierra Belén - Cat. & $\mathrm{Ar} / \mathrm{K}$ & biotite & $386 \pm 4$ & 1 & $(*)$ \\
\hline$A N(A L) 2$ & Granite - El Alto - S. Ancasti & $\mathrm{Ar} / \mathrm{K}$ & biotite & $398 \pm 15$ & 2 & $(*)$ \\
\hline LP-CI & Microtonalite - Sierra de Macha, Cba & $\mathrm{Ar} / \mathrm{K}$ & whole rock & $399 \pm 6$ & 1 & 5 \\
\hline LO- 2 & Granite-Loma Pelada, Cumbres Calchaquies, & onn $\mathrm{non}$ & & & 1 & $(*)$ \\
\hline
\end{tabular}


Table $1-$ Radiometric ages of the igneous rocks

(continuaşão)

\begin{tabular}{|c|c|c|c|c|c|c|}
\hline Sample & - Description and Locality & Method & Mineral & Age in m.y. & Laboratory & Reference \\
\hline 134 & Porphyritic granite - Villa Praga, San Luis & $\mathrm{Sr} / \mathrm{Rb}$ & whole rock & $415 \pm 25$ & 4 & 2 \\
\hline 140 & Porphyritic granite - Renca, San Luis & $\mathrm{Sr} / \mathrm{Rb}$ & whole rock & $415 \pm 25$ & 4 & 2 \\
\hline 141 & Granite - Cantera Pelia Pintada. San Luis & $\mathrm{Sr} / \mathrm{Rb}$ & whole rock & $415 \pm 25$ & 4 & 2 \\
\hline LN-10 & Tonalite Los Pocitos $-S$ de Los Llanos, & & & & & \\
\hline$\because$ & La Rioja & $\mathrm{A}: \mathrm{r} / \mathrm{K}$ & biotite & $423 \pm 22$ & 1 & $(*)$ \\
\hline$V F(S A) 7$ & Granodiorite - San Agustín - S. Valle Fértil & $\mathrm{Ar} / \mathrm{K}$ & biotite & $430 \pm 15$ & 2 & $(*)$ \\
\hline LP-33 & Microtonalite - S. de Macha, Cba & $\mathrm{Ar} / \mathrm{K}$ & whole rock & $439 \pm 21$ & 1 & 5 \\
\hline LP-32 & Microtonalste-S. de Macha, Cba & $\mathrm{Ar} / \mathrm{K}$ & whole rock & $440 \pm 21$ & 1 & 5 \\
\hline \multirow[t]{2}{*}{$L N-21$} & Granodiorite -... Villa Casana, S de Chepes, & & & & & \\
\hline & La Rjoja & $\mathrm{Ar} / \mathrm{K}$ & biotite & $440 \pm 7$ & 1 & $(*)$ \\
\hline LN-2 & Tonalite - Ofpas, S. de Los Llanos, La Rioja & $\mathrm{Ar} / \mathrm{K}$ & biotite & $441 \pm 23$ & 1 & $(*)$ \\
\hline$V-1$ & Porphyritic granite - Cuesta de Zapata & $\mathrm{Ar} / \mathrm{K}$ & biotite & $444 \pm 6$ & 2 & $(*)$ \\
\hline \multirow[t]{2}{*}{$\mathrm{MAG}$} & Granodiorite - Cuesta de Miranda - S & & & & & \\
\hline & Famatina & $\mathrm{Ar} / \mathrm{K}$ & biotite & $448 \pm 20$ & (1) & $(*)$ \\
\hline \multirow[t]{2}{*}{ LZ-005 } & Diorite -.. Napa between Charbonier and & & & & & \\
\hline & Ischiłin, Cba & $\mathrm{Pb} / \mathrm{a}$ & zircon & $450 \pm 50$ & 3 & 4 \\
\hline \multirow[t]{2}{*}{ LZ-005 } & Diorite - Napa between Charbonier and & & & & & \\
\hline & Ischilin, Cba & $\mathrm{Ar} / \mathrm{K}$ & biotite & $452 \pm 20$ & 2 & 4 \\
\hline S $3-4$ & Andesite - S. Ambargasta, S. del Estero & $\mathrm{Ar} / \mathrm{K}$ & whole rock & $452 \pm 6$ & 1 & 6 \\
\hline \multirow[t]{2}{*}{$\mathrm{LN}-23$} & Tonalite - San Antonio, S. de Chepes, La & & & & & \\
\hline & Rioja & $\mathrm{Ar} / \mathrm{K}$ & biotite & $453 \pm 24$ & 1 & $\left({ }^{*}\right)$ \\
\hline \multirow[t]{2}{*}{ TV-4S } & Granite-Mala-Mala, Cumbres Calchaquíes, & & & & & \\
\hline & Tuc. & $\mathrm{Ar} / \mathrm{K}$ & biotite & $456 \pm 21$ & 1 & 6 \\
\hline $\mathrm{H}(\mathrm{LT}-1)$ & Granodiorite -. Las Tumanas - San Juan & $\mathrm{Ar} / \mathrm{K}$ & biotite & $456 \pm 15$ & 2 & $(*)$ \\
\hline SJ-5 & Andesite-S de Ambargasta, S. del Estero & $\mathrm{Ar} / \mathrm{K}$ & whole rock & $461 \pm 22$ & 1 & 6 \\
\hline SJ -4 & Andesite-S. de Ambargasta, S. del Estero & $\mathrm{Ar} / \mathrm{K}$ & whole rock & $461 \pm 22$ & 1 & 6 \\
\hline LU-001 & Pegmatite - Sta. Ana, Pringles, Sn Luis & $\mathrm{Pb} / \mathrm{Ur}$ & uraninite & $465 \pm 15$ & 3 & 4 \\
\hline 44 & Porphyritic granite - La Higuerita, Cba & $\mathrm{Pb} / \mathrm{a}$ & & $465 \pm 50$ & 1 & 3 \\
\hline \multirow[t]{2}{*}{$\mathbf{R} \mathbf{2} \sim 2$} & Granite - Rio Sosa, Cumbres Calchaquies, & & & & & \\
\hline & Tuc. & $\mathrm{Ar} / \mathrm{K}$ & biotite & $466 \pm 10$ & 1 & 6 \\
\hline LI-2 & Tonalite - La Isabella, Cba & $\mathrm{Ar} / \mathrm{K}$ & biotite & $467 \pm 22$ & 1 & 5 \\
\hline $\mathrm{LN}-14$ & Granodiorite --Atiles, Malanzán, La Rioja & $\mathrm{Ar} / \mathrm{K}$ & biotite & $468 \pm 12$ & 1 & $(*)$ \\
\hline \multirow[t]{2}{*}{ RS -1} & Granite - Rio Sosa, Cumbres Calchaquies, & & & & & \\
\hline & Tuc. & $\mathrm{Ar} / \mathrm{K}$ & biolite & $471 \pm 9$ & 1 & 6 \\
\hline $\mathrm{LU}-00 \mathrm{~S}$ & Pegmalite-Qda de] Tigre, San Javier, Cba & $\mathrm{Pb} / \mathrm{UQ}$ & uraninité & $472 \pm 25$ & 3 & 4 \\
\hline \multirow[t]{2}{*}{ TV-44 } & Granite-Mala-Mala, Cumbres Calchaquies, & & . & & & \\
\hline & Tuc. & $\mathrm{Ar} / \mathrm{K}$ & biotite & $473 \pm 22$ & 1 & 6 \\
\hline \multirow{2}{*}{$\begin{array}{l}\text { LU.004 } \\
\text { TV.39 }\end{array}$} & Pegmalite - Cerro Blanco, San Javier, Cba & $\mathrm{Pb} / \mathrm{UQ}$ & uraninitec & $477 \pm 25$ & 3 & 4 \\
\hline & $\begin{array}{l}\text { Granite - Mala-Mala, Cumbres Calchaquies, } \\
\text { Cba }\end{array}$ & $\mathrm{Ar} / \mathrm{K}$ & biotite & $479+9$ & 1 & 6 \\
\hline SJ-5 & Andesite - S. de Ambargasta, S. del Estero & $\mathrm{Ar} / \mathrm{K}$ & whole rock & $479 \pm 8$ & 1 & 5 \\
\hline LU-006 & Pegmatite - Angel, San Javier, Cba & $\mathrm{Pb} / \mathrm{U} \mathrm{I}$ & uraninite & $480 \pm 15$ & 3 & 4 \\
\hline LU-002 & Pegmatite - La Elsa, San Javier, Cba & $\mathrm{Pb} / \mathrm{U} \mathrm{I}$ & uraninite & $490 \pm 15$ & 3 & 4 \\
\hline $133 \mathrm{D}$ & Turmalinic granile - Villa Praga, San Luis & $\mathrm{Sr} / \mathrm{Rb}$ & & $500 \pm 20$ & 4 & 2 \\
\hline 137 & Granite with garnet - Villa Praga, San Luis & $\mathrm{Sr} / \mathrm{Rb}$ & & $500 \pm 20$ & 4 & 2 \\
\hline LZ-004 & Porphyritic granife - Ischilin, Cba & $\mathrm{Pb} / \mathrm{a}$ & zircon & $500 \pm 50$ & 3 & 4 \\
\hline AMB 5 & Granite - Santa Maria - S. Ambargasta & $\mathrm{Ar} / \mathrm{K}$ & biotite & $500 \pm 15$ & 2 & $(*)$ \\
\hline AMB-2 & Granite - Chuña Huasi - S. Ambargasta & $\mathrm{Ar} / \mathrm{K}$ & biotile & $517 \pm 15$ & 2 & $(*)$ \\
\hline LZ-054 & Granodiorite - Cruz del Eje, Cba & $\mathrm{Ar} / \mathrm{K}$ & muscovite & $520 \pm 20$ & 2 & 4 \\
\hline $\mathbf{L U} \cdot 003$ & Pegmatite $\cdots$ Las Tapias, Cba & $\mathrm{Pb} / \mathrm{UI}$ & uraninite & $520 \pm 20$ & 3 & 4 \\
\hline $\mathrm{G}-1$ & Granite - Guasayán, S. del Estero & $\mathrm{Ar} / \mathrm{K}$ & biotite & $541 \pm 8$ & 1 & $(*)$ \\
\hline LI-1 & Diorite - La Isabella, S de Macha, Cba & $\mathrm{Ar} / \mathrm{K}$ & anfibol & $557 \pm 26$ & 1 & 5 \\
\hline
\end{tabular}

\section{REFER ENCES}

1. Centro de Pesquisas Geocronologicas of the University of São Paulo.

2. Instituto de Geocronologia y Geología Isotópica, Buenos Aires.

3. División Estudios especiales, Gerencia materia prima (CNEA).

4. Yale University U.S.A.

5. Academy National of Sciences of Leningrado, USSR.

6. Cambridge University, England.
(1) Kittl (1965)

(2) Halpern-Linares-La Turre (1970)

(3) Stipanicic-Linares (1969)

(4) Linares-La Torre (1969)

(5) González-Toselli (1972)

(6) González (1972)

(*) inéditos 
In Fig. 1, three concentric age provinces were established, the boundaries of which determine the following areas:

a) Two external areas with the older rocks. The eastern belt occupies the Cumbres Calchaquíes (Province of Tucumán), Sierras de Guasayán and Amabargasta (Province of Santiago del Estero) and Sierra de Macha and some bodies in Sierra Chica (Province of Córdoba). The western belt includes the Sierra de Chepes and Sierras de Los Llanos and Malanzán (Province of La Rioja), and Sierras de Valle Fértil and La Huerta (Province of San Juan). It extends to the Province of San Luis.

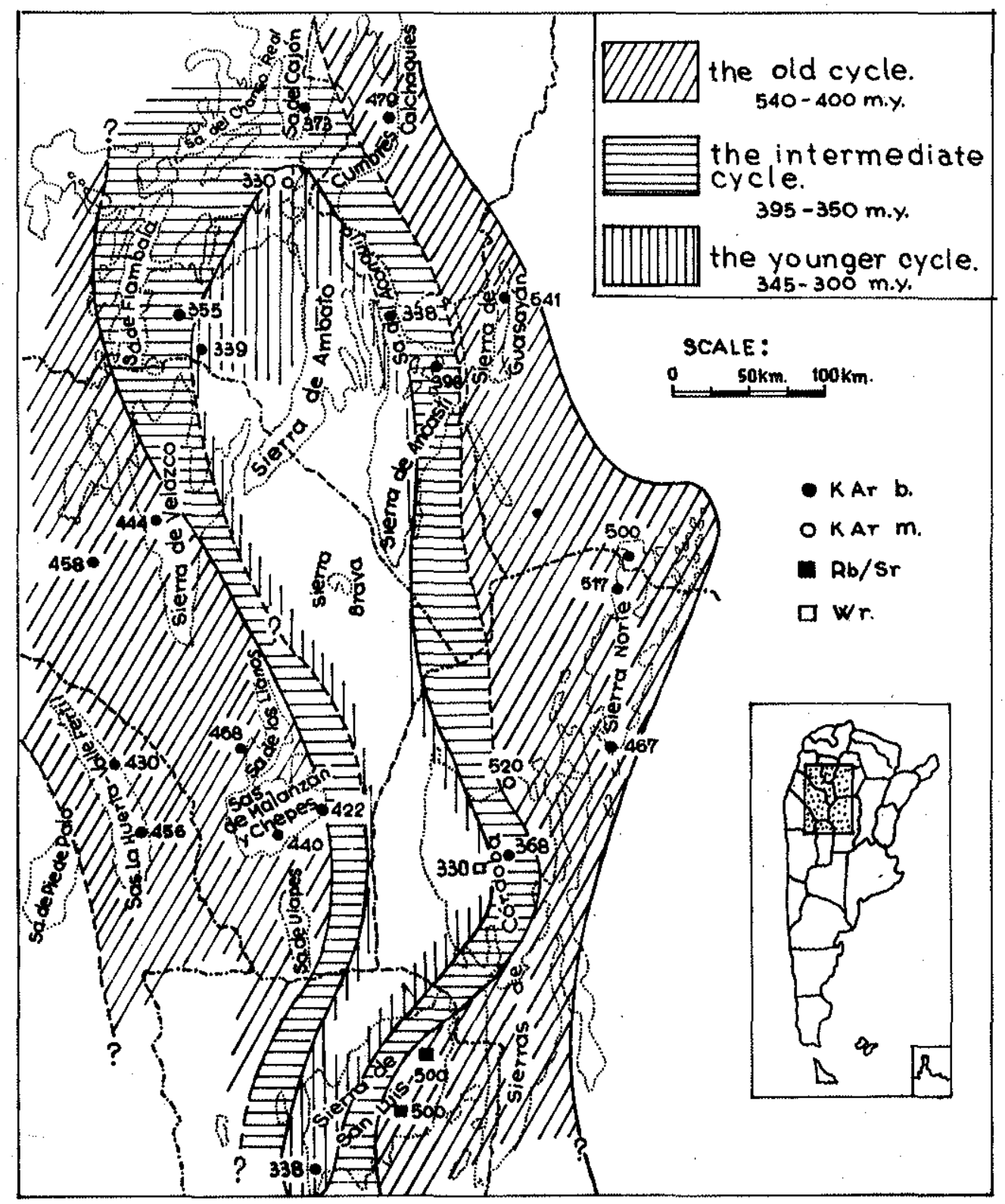

Figure 1 - Concentric age belts in the Sierras Pampeanas. Tentative plan. Only some of the data appearing in Table $\mathrm{I}$ are indicated 
b) An internal area with the younger rocks: the plutons of Los Pinos and San Ignacio (Province of Tucumán) in the north, and those of La Toma and Los Cóndores (Province of San Luis) in the south; between them, the great batholith of Achala (Province of Córdoba).

c) Two intermediate areas, including the western side of Sierra de Aconquija (Prom vince of Tucumán), Sierras de Belén and Zapata (Province of Catamarca) and a part of Sierra Chica (Province of Córdoba).

CONCLUSIONS 1) According to the radiometric dates made to the present, there is no indication of magmatic activity during Precambrian times in the Sierras Pampeanas.

2) The threefold subdivision suggested by Linares and La Torre (1969) for Paleozoic magmatic cycles is confirmed.

3) The limits referred by these authors can be modified as follows: a) the older cycle: 540-400 m.y.; b) the intermediate one: 395-350 m.y.; c) the younger one: $345-300$ m.y.

4) The corresponding magmatic rocks outcrop in three concentric belts, the boundaries of which can not always be well defined by the present lack of additional radiometric ages.

Acknowledgements The authors acknowledge the contributions of Dr. U. Cordani and K. Kawashita from the Centro de Pesquisas Geocronologicas of São Paulo (Brazil), who helped during the analytical work at São Paulo; of. Dr. Enriquue Linares from Instituto de Geocronología y Geología Isotópica of Buenos Aires, where additional radiometric ages were obtained, and of Dr. Manuel Cábrera for his personal contribution.

\section{REFERENCES}

BONARELli, G. and PASTORE, F. - 1918 - Bosquejo geologico de la Provincia de Tucumán, 1." Reunión Nac. Soc. Argentina Ciencias Naturales. Tucumán, pp. 27-46

CAELLES, N. - 1971 - Potassium-argon ages of porphyry copper deposits and associated rocks in the Farallon Negro-Capillitas district, Gatamarca, Argentina, Economic Geology, 46: 961-964

GONZÁLEZ BONORINO, F. - 1950 - Algunos problemas geológicos de las Sierras Pampeanas, Rev. Asoc. Geol. Argentina, V (3): 81-110

GONZÁLEZ, R. - 1971 - Edades radimétricas de algunos cuerpos eruptivos de Argentina, Rev. Asoc. Geol. Argentina, XXVI (3): 411

GONZALEZ, R., KAWASHITA, K. and CABRERA, M. - 1971 - Edades radimétricas de algunas rocas del basamento de las Sierras Pampeanas, Rev. Asoc. Geol. Argentina, XXVI (4): 527-528

GONZALEZ, R., TOSELLI, A. and CABRERA, M. - 1972 - Las intrusiones diorítico-tonaliticas de Sierra de Macha (provincia de Córdoba), Acta Geol. Lilloana, XII (2): 11-12

HARRINGTON, H. H. - 1956 - Argentina, in Handbook of South American Geology, Geol. Soc. America, Memoir 65, pp. 1773-1814

HALPERN, M., LINARES, E. and LA TORRE, C. - 1970 - Estudio preliminar por el método estroncio-rubidio de rocas metamórficas y graníticas de la provincia de San Luis, República Argentina, Rev. Asoc. Geol. Argentina, XXV (3): 293-302

KITTL, E. - 1965 - La edad de las rocas graníticas y los ciclos metalogenéticos de Argentina, Bol. Acad. Nac. Ciencias Córdoba, XLIV: 97-104

LINARES, E. - 1968 - Datación geologica de las rocas graníticas de las sierras de Córdoba por medio del método plomo-alfa, Actas 3as. Jornadas Geol. Argentinas, II : 199-217

LINARES, E. and LA TORRE, C. - 1969 - Edades potasio-argón y plomo-alfa de rocas graníticas de las Provincias de Córdoba y San Luis, Actas $4 .^{\circ}$ Congr. Geológico Argentino, II: 195-204

RASSMUSS, J. - 1918 - La Sierra de Aconquija, 1. Reunión Nacional Soc. Argentina de Ciencias Naturales, Tucumán, pp. 47-69

STIPANICIC, P. and LINARES, E. - 1969 - Edades radimétricas determinadas para la República Argentina y su significado geológico, Bol. Acad. Nac. Ciencias Córdoba, XLVII: 51-96 